UDC: 517.925 .4

45 (LXXI) No.2 (2021), 85-93

DOI: $10.37560 /$ matbil $21452085 z$

\title{
ON THE INTEGRABILITY OF A CLASS OF DIFFERENTIAL EQUATIONS
}

\author{
BILJANA ZLATANOVSKA ${ }^{1}$ AND BORO M. PIPEREVSKI ${ }^{2}$
}

\begin{abstract}
In this paper, a class of second-order linear differential equations is reviewed. For this class of B.S.Popov necessary and sufficient condition for reductable according to Frobenius is obtained. By using another method, the same condition is obtained where the existence of the natural number $n$ is replaced by the existence of an integer n. For the same class of second-order linear differential equations, the case for reductable according to Frobenius which is independent from an exist of a number $n$ is reviewed. In both cases, formulas of one particular solution and transformation to a system of firstorder differential equations are obtained. In end, this theory is supported by examples.
\end{abstract}

\section{INTRODUCTION}

In [1, a class of second-order linear differential equations

$$
\ddot{z}+\left(\alpha e^{t}+\beta\right) \dot{z}+\left(A_{1} e^{2 t}+B_{1} e^{t}+C_{1}\right) z=0, \quad \alpha, \beta, A_{1}, B_{1}, C_{1} \in \mathbb{R}
$$

is reviewed.

For the equation (1.1), the following necessary and sufficient condition for reductable according to Frobenius is obtained by the method of Mitrinovic.

The equation (1.1) is reductable according to Frobenius if and only if there exists a natural number $\mathrm{n}$ that satisfies the condition

$$
\alpha \beta-2 B_{1}+\alpha=\mp \sqrt{\alpha^{2}-4 A_{1}}\left[2 n+1-\sqrt{\beta^{2}-4 C_{1}}\right]
$$

In this paper for this class of second-order linear differential equations using another method the same condition is obtained by what the existence of a natural number $\mathrm{n}$ is replaced by the existence of an integer $\mathrm{n}$. This is the coverage of a larger class of differential equations. For the same class of second-order linear differential equations, the case for reductable according to Frobenius which is

2010 Mathematics Subject Classification. 34A05, 34A30.

Key words and phrases. Second-order linear differential equations, system of first-order differential equations, particular solution.

Dedicated to the Day of Differential Equations in Macedonia 2021. 
independent from an exist of a number $\mathrm{n}$ is reviewed. In both cases, formulas of one particular solution and transformation to a system of first-order differential equations are obtained.

In the mathematical literature [2, 3, 4, the following theorems and a definition that are known are:

Theorem 1. Let the differential equation

$$
\left(A x^{2}+B x+C\right) y^{\prime \prime}+(D x+E) y^{\prime}+F y=0, \quad A, B, C, D, E, F \in \mathbb{R}
$$

is given. The differential equation (1.3) is integrable if there exists an integer $n \in Z$ (the smallest number after absolute value if there are such numbers) that satisfies the condition

$$
n(n-1) A+n D+F=0
$$

In doing so, the differential equation (1.3) has a particular solution which is given by the formula

$y_{p}=P_{n}(x)=\left(A x^{2}+B x+C\right) e^{-\int \frac{(D x+E)}{\left(A x^{2}+B x+C\right)} d x}\left[\left(A x^{2}+B x+C\right)^{n-1} e^{\int \frac{(D x+E)}{\left(A x^{2}+B x+C\right)} d x}\right]^{(n)}$

if $n \in \mathbb{N}$ (a polynomial solution). But, if $n \in \mathbb{Z}^{-}, k=-(n+1) \in \mathbb{N}$ then a particular solution will be given by the formula

$$
y_{p}(x)=\left[\left(A x^{2}+B x+C\right)^{k+1} e^{-\int \frac{(D x+E)}{\left(A x^{2}+B x+C\right)} d x}\right]^{(k)}
$$

It is well known that if a second-order linear homogeneous differential equation is integrable then it is also reductable according to Frobenius.

Theorem 2. Let the differential equation

$$
P(x) y^{\prime \prime}+Q(x) y^{\prime}+R(x)=0
$$

is given. Let the equation (1.7) has a particular solution $F(x)$. Then the equation (1.7) is reductable according to Frobenius and the equation (1.7) is transformed to the system of first-order differential equations

$$
\begin{array}{r}
F(x) y^{\prime}-F^{\prime}(x) y=z \\
P(x) z^{\prime}+Q(x) z=0
\end{array}
$$

Definition 1. (Frobenius): A linear homogeneous differential equation whose coefficients are unambiguous functions is called more predictable according to Frobenius if there is no common solution with a linear homogeneous differential equation with coefficients unambiguous lower order functions. Otherwise it is called reductable according to Frobenius.

This theory is supported by other mathematical papers such as [5, 6, 7].

\section{EXTENDing of the Class of DifFERENTIAL EQUATIONS}

For the differential equation (1.1) there exists a natural number $n$, which satisfies the condition $(1.2)$. For the same differential equation $(1.1)$, we replace the existence of the natural number $\mathrm{n}$ with the existence of an integer $n$ with the following Theorem 3 . 
Theorem 3. The differential equation

$$
\ddot{z}+\left(\alpha e^{t}+\beta\right) \dot{z}+\left(A_{1} e^{2 t}+B_{1} e^{t}+C_{1}\right) z=0, \quad \alpha, \beta, A_{1}, B_{1}, C_{1} \in \mathbb{R}
$$

is integrable if exist $n \in Z$ which satisfies the condition

$$
2 B_{1}-\alpha \beta-\alpha+(2 n+1)\left[ \pm \sqrt{\alpha^{2}-4 A_{1}}\right]+\left[ \pm \sqrt{\alpha^{2}-4 A_{1}}\right]\left[ \pm \sqrt{\beta^{2}-4 C_{1}}\right]=0
$$

Proof. Let us consider the differential equation

$$
x y^{\prime \prime}+(D x+E) y^{\prime}+F y=0, \quad D, E, F \in \mathbb{R}
$$

where

By the substitution

$$
y=y(x), \quad y^{\prime}=\frac{d y}{d x}, \quad y^{\prime \prime}=\frac{d^{2} y}{d x^{2}}
$$

$$
x=e^{t}
$$

the differential equation (2.1) is transformed in the differential equation

$$
\ddot{y}+\left[D e^{t}+E-1\right] \dot{y}+F e^{t} y=0
$$

where

By the substitution

$$
y=y(x), \quad \dot{y}=\frac{d y}{d t}, \quad \ddot{y}=\frac{d^{2} y}{d t^{2}}
$$

$$
y(t)=e^{\int\left(r e^{t}+s\right) d t} z(t), \quad r, s \in \mathbb{R}
$$

the differential equation 2.5 is transformed in the differential equation

$\ddot{z}+\left[(2 r+D) e^{t}+2 s+E-1\right] \dot{z}+\left[\left(r^{2}+r D\right) e^{2 t}+(2 r s+r E+s D+F) e^{t}+s^{2}+s E-s\right] z=0$

where

$$
z=z(x), \quad \dot{z}=\frac{d z}{d t}, \quad \ddot{z}=\frac{d^{2} z}{d t^{2}}
$$

The equation (2.1) is equal of the equation 2.7 if the following relations

$$
\begin{aligned}
2 r+D & =\alpha \\
2 s+E-1 & =\beta \\
r^{2}+r D & =A_{1} \\
2 r s+r E+s D+F & =B_{1} \\
s^{2}+s E-s & =C_{1}
\end{aligned}
$$

are satisfied. 
From the relations 2.8 , the relations

$$
\begin{aligned}
D & = \pm \sqrt{\alpha^{2}-4 A_{1}}, \quad E=1 \pm \sqrt{\beta^{2}-4 C_{1}} \\
r & =-\frac{1}{2}\left(-\alpha \pm \sqrt{\alpha^{2}-4 A_{1}}\right), \quad s=-\frac{1}{2}\left(-\beta \pm \sqrt{\beta^{2}-4 C_{1}}\right) \\
F & =\frac{1}{2}\left[2 B_{1}-\alpha-\alpha \beta+\left[ \pm \sqrt{\alpha^{2}-4 A_{1}}\right]+\left[ \pm \sqrt{\alpha^{2}-4 A_{1}}\right]\left[ \pm \sqrt{\beta^{2}-4 C_{1}}\right]\right]
\end{aligned}
$$

are obtained.

By using the Theorem 1 the equation 2.3 is integrable if there exists an integer $n \in \mathbb{Z}$ that satisfies the condition

$$
n D+F=0
$$

By using the relations $\sqrt{2.9}$ in the condition $\sqrt{2.10}$ is obtained the relation $(2.2)$.

Remark 1: In connections 2.9 the sign before the roots is equal to the sign before the roots the condition $(2.2)$.

By the existence of an integer $n$, which satisfies the condition 2.10 of Theorem 1. we have made an extension of the class of differential equations (2.1). This covers a larger number of differential equations that the differential equations given in [1].

On particular integral can be computed for the class of differential equations (2.1). The formulas for calculating the particular integral are given by the following Theorem 4 .

Theorem 4. Let the differential equation (2.1) is given. Let the condition (2.2) is satisfied. Then one the particular solution is given by the formula

$$
z_{p}(t)=e^{-\int\left(r e^{t}+s\right) d t} y_{p}\left(e^{t}\right)
$$

where

$$
y_{p}(x)=P_{n}(x)=x^{1-E} e^{-D x}\left[x^{n-1+E} e^{D x}\right]^{(n)}
$$

if $n \in \mathbb{N}$,

or

$$
y_{p}(x)=\left[x^{k+1-E} e^{-D x}\right]^{(k)}
$$

if $n \in \mathbb{Z}^{-}, k=-(n+1) \in \mathbb{N}$.

By using the formulas (2.9) according to the Remark 1, the coefficients $r, s, D, E$ are given.

Proof. By using the formulas $(1.5)$ and $(1.6)$ from Theorem 1 in the equation (2.3), the formulas 2.12 and $(2.13)$ are obtained. Finally, by using the substitutions (2.4) and (2.6), the formula (2.11) is obtained.

The differential equation (2.1) can be transformed in the system of differential equations. This is given by Theorem 5 . 
Theorem 5. Let the differential equation (2.1) is given. Let the condition (2.2) is satisfied. Then the equation (2.1) is reductable and the equation (2.1) is transformed to the system of first-order differential equation

$$
\begin{aligned}
z_{p}(t) z^{\prime}-z_{p}^{\prime}(t) z & =w \\
w^{\prime}+\left(\alpha e^{t}+\beta\right) w & =0
\end{aligned}
$$

where $z_{p}(t)$ is a particular solution of the differential equation (2.1).

Proof. By using of Theorem 2 the differential equation (2.1) is reductable and the differential equation (2.1) is transformed to the system 2.14).

Next, the case for reductable according to Frobenius of the differential equation (2.1), which is independent from an exist of a number $\mathrm{n}$ is reviewed. The proving procedure in this case is the same as in the case where reductable according to Frobenius of the differential equation (2.1) depends on the existence of $n$ given by the previous Theorem 3 . Theorem 4 and Theorem 5 . For this case we have the following Lemma 1

Lemma 1. The differential equation (2.1) is reductable according to Frobenius if the conditions

$$
\begin{aligned}
\alpha^{2}-4 A_{1} & =0 \\
2 B_{1}-\alpha \beta-\alpha & =0
\end{aligned}
$$

are satisfied.

By the formula

$$
z_{p}(t)=e^{-\int\left(r e^{t}+s\right) d t}
$$

a particular solution is given.

The differential equation (2.1) is transformed to the system of first-order differential equation (2.14) where

$$
r=\frac{\alpha}{2}, \quad s=\frac{1}{2}\left(\beta \pm \sqrt{\beta^{2}-4 C_{1}}\right)
$$

Proof. Let us consider the differential equation

$$
x y^{\prime \prime}+E y^{\prime}=0
$$

where

$$
y=y(x), \quad y^{\prime}=\frac{d y}{d x}, \quad y^{\prime \prime}=\frac{d^{2} y}{d x^{2}}
$$

By the substitution 2.4, the differential equation 2.1 can be writen as

$$
\ddot{y}+[E-1] \dot{y}=0
$$

where

$$
y=y(t), \quad \dot{y}=\frac{d y}{d t}, \quad \ddot{y}=\frac{d^{2} y}{d t^{2}}
$$

By the subsitution (2.6), this differential equation is transformed in the differential equation

$$
\ddot{z}+\left[2 r e^{t}+2 s+E-1\right] \dot{z}+\left[r^{2} e^{2 t}+(2 r s+r E) e^{t}+s^{2}+s E-s\right] z=0
$$


where

$$
z=z(t), \quad \dot{z}=\frac{d z}{d t}, \quad \ddot{z}=\frac{d^{2} z}{d t^{2}}
$$

The equation (2.1) is equal of the equation 2.18 if the following relations

$$
\begin{aligned}
& 2 r=\alpha \\
& 2 s+E-1=\beta \\
& r^{2}=A_{1} \\
& 2 r s+r E=B_{1} \\
& s^{2}+s E-s=C_{1}
\end{aligned}
$$

are satisfied.

From these relations, the formulas

$$
E=1 \mp \sqrt{\beta^{2}-4 C_{1}}, \quad r=\frac{\alpha}{2}, \quad s=\frac{1}{2}\left(\beta \pm \sqrt{\left.\beta^{2}-4 C_{1}\right)}\right.
$$

and the conditions (2.15) are obtained.

The differential equation (2.17) has a particular solution $y=K$ ( $K$ is a constant). By the substitution (2.6), the formula (2.16) is obtained. By applied of Theorem 2 the differential equation $(2.1)$ is transformed to the system of first-order differential equation (2.14).

\section{EXAmples of the DifFerential EQUATIONS}

In this section, we will give examples of differential equations belonging to the extended class of differential equations from Theorem 3 . Using Theorem 4, we will compute a particular integral for a given differential equation.

Example 1. Let the differential equation

$$
\ddot{z}+\left(3 e^{t}-2\right) \dot{z}+\left(2 e^{2 t}+1\right) z=0
$$

is given.

A differential equation (3.1) satisfies the condition 2.2 for $n=-2$. By using the formulas 2.9 according the Remark 1 the coefficients $r=1, s=-1, D=1, E=1$ are obtained. According to the formula 2.13 for $k=1$, the formula

$$
y_{p}(x)=e^{-x}(1-x)
$$

is obtained.

But, by the formula (2.11), the particular solution of the differential equation (3.1)

$$
z_{p}(x)=e^{-2 e^{t}}\left(e^{t}-e^{2 t}\right)
$$

is obtained.

By using the Theorem 5 the differential equation (3.1) can transform to the following system of first-order differential equation

$$
\begin{aligned}
& z_{p}(t) z^{\prime}-z_{p}^{\prime}(t) z=w \\
& w^{\prime}+\left(3 e^{t}-2\right) w=0
\end{aligned}
$$

where $z_{p}(t)$ is a particular solution which is given by the formula 3.2 . 
Example 2. Let the differential equation

$$
\ddot{z}-\left(5 e^{t}+5\right) \dot{z}+\left(4 e^{2 t}+e^{t}+4\right) z=0
$$

is given.

A differential equation (3.3) satisfies the condition 2.2 for $n=-2$. By using the formulas 2.9. according the Remark 1 the coefficients $r=-1, s=-1, \quad D=-3$, $E=-2$ are obtained. According to the formula $\sqrt{2.13}$ for $k=1$, the formula

$$
y_{p}(x)=x^{3} e^{3 x}(4+3 x)
$$

is obtained.

But, by the formula (2.11), the particular solution of the differential equation (3.3)

$$
z_{p}(x)=e^{4 e^{t}}\left(4 e^{4 t}+3 e^{5 t}\right)
$$

is obtained.

By using the Theorem 5 the differential equation 3.3 can transform to the following system of first-order differential equation

$$
\begin{aligned}
& z_{p}(t) z^{\prime}-z_{p}^{\prime}(t) z=w \\
& w^{\prime}-\left(5 e^{t}+5\right) w=0
\end{aligned}
$$

where $z_{p}(t)$ is a particular solution which is given by the formula (3.4).

Example 3. Let the differential equation

$$
\ddot{z}+\left(-e^{t}+2\right) \dot{z}+2 e^{t} z=0
$$

is given.

A differential equation (3.5) satisfies the condition $(2.2)$ for $n=-3$. By using the formulas (2.9) according to Remark 1 the coefficients $r=-1, s=2, D=1$, $E=-1$ are obtained. According to the formula (2.13) for $k=2$, the formula

$$
y_{p}(x)=e^{-x}\left(x^{4}-8 x^{3}+12 x^{2}\right)
$$

is obtained.

But, by the formula (2.11), the particular solution of the differential equation (3.5)

$$
z_{p}(x)=e^{2 t}-8 e^{t}+12
$$

is obtained.

By using the Theorem 5 the differential equation 3.5 can transform to the following system of first-order differential equation

$$
\begin{gathered}
z_{p}(t) z^{\prime}-z_{p}^{\prime}(t) z=w \\
w^{\prime}+\left(-e^{t}+2\right) w=0
\end{gathered}
$$

where $z_{p}(t)$ is a particular solution which is given by the formula 3.6 .

Next examples will be solved with applied of Lemma 1

Example 4. Let the differential equation

$$
\ddot{z}+\left(2 e^{t}+1\right) \dot{z}+\left(e^{2 t}+2 e^{t}-2\right) z=0
$$

is given. 
A differential equation (3.7) satisfies the condition 2.15 from the Lemma 1 By using the formula (2.16), a particular solution of the differential equation (3.7)

$$
z_{p}(x)=e^{-e^{t}} e^{t}
$$

is obtained. The differential equation (3.7) can transform to the following system of first-order differential equation

$$
\begin{aligned}
& z_{p}(t) z^{\prime}-z_{p}^{\prime}(t) z=w \\
& w^{\prime}+\left(2 e^{t}+1\right) w=0
\end{aligned}
$$

according to 2.14 .

Example 5. Let the differential equation

$$
\ddot{z}+\left(2 e^{t}+2\right) \dot{z}+\left(e^{2 t}+3 e^{t}+1\right) z=0
$$

is given.

A differential equation (3.8) satisfies the condition 2.15 from the Lemma 1 . By using the formula (2.16), a particular solution of the differential equation 3.7)

$$
z_{p}(x)=e^{-e^{t}} e^{-t}
$$

is obtained. The differential equation (3.8) can transform to the following system of first-order differential equation

$$
\begin{aligned}
& z_{p}(t) z^{\prime}-z_{p}^{\prime}(t) z=w \\
& w^{\prime}+\left(2 e^{t}+2\right) w=0
\end{aligned}
$$

according to 2.15 .

\section{REFERENCES}

[1] S. B. Popov, Forming of reductability criteria for some classes of linear differential equations, Annual Proceedings of the Faculty of Philosophy, University of Skopje, Department of Natural Sciences and Mathematics, Book $5 N^{0} 2$ (1952), 1-68.

[2] I. A. Shapkarev, B. M. Piperevski, E. I. Hadzieva, N. Serafimova, K. Mitkovska-Trendova, About a class of second order differential equations, whose general solution is polynomial, Seventh Macedonian Symposium on Differential Equations, Proceedings of Papers, 2002, 27-40. http://www.cim.feit.ukim.edu.mk

[3] B. M. Piperevski, N. Serafimova, Existence and construction of the general solution of a class of second order differential equations with polynomial coefficients, Seventh Macedonian Symposium on Differential Equations, Proceedings of Papers, 2002, 41-52. http://www.cim.feit.ukim.edu.mk

[4] B. M. Piperevski, B. Zlatanovska, About one B.S. Popov's result, Balkan Journal of applied mathematics and informatics (BJAMI), 3(2) (2020), 15-23.

[5] S. B. Popov, On the reductability of the hypergeometric differential equation, Annual Proceedings of the Faculty of Philosophy of University of Skopje, Book $4 N^{0} 7$, (1951), 1-20.

[6] G. Frobenius, Ueber den Begriff der Irreductibilitat der Theorie der linearen Differentialgleichungen, Journal für die reine und angewandte Mathematik, T.76. (1878), 236-271.

[7] E. Picard, Traite d'analyse, t. III, Deuxieme edition (1908), 560-561. 
1 Goce Delcev University,

Faculty of Computer Science,

Krste Misirkov 10-A, Stip, North Macedonia

Email address: biljana.zlatanovska@ugd.edu.mk

2 Ss. Cyril and Methodius University,

Faculty of Electrical Engineering and Information Technologies,

Ruger Boskovik 18, Skopje, North Macedonia

Email address: borom@feit.ukim.edu.mk

Received: 17.5 .2021

Revised: 26.5 .2021

Accepted: 26.5.2021 